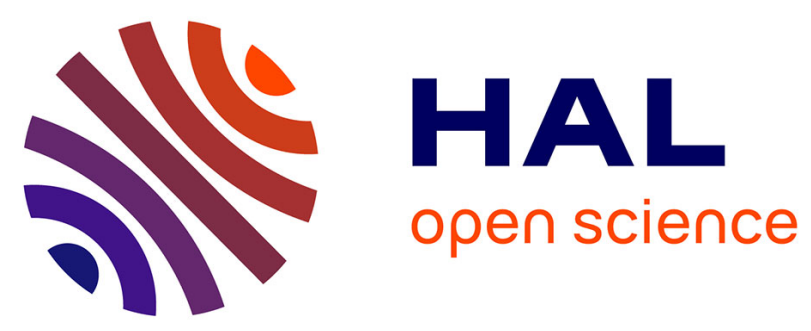

\title{
A Time-Of-Flight Gamma Camera Data Acquisition System for Hadrontherapy Monitoring
}

X. Chen, O. Allegrini, B. Carlus, C. Caplan, L. Caponetto, J. P. Cachemiche, S. Curtoni, D. Dauvergne, R. Della Negra, M. Fontana, et al.

\section{- To cite this version:}

X. Chen, O. Allegrini, B. Carlus, C. Caplan, L. Caponetto, et al.. A Time-Of-Flight Gamma Camera Data Acquisition System for Hadrontherapy Monitoring. 2019 IEEE Nuclear Science Symposium (NSS) and Medical Imaging Conference (MIC), Oct 2019, Manchester, United Kingdom. pp.1-3, 10.1109/NSS/MIC42101.2019.9060072 . hal-02309735

\section{HAL Id: hal-02309735 \\ https://hal.science/hal-02309735}

Submitted on 20 Mar 2020

HAL is a multi-disciplinary open access archive for the deposit and dissemination of scientific research documents, whether they are published or not. The documents may come from teaching and research institutions in France or abroad, or from public or private research centers.
L'archive ouverte pluridisciplinaire HAL, est destinée au dépôt et à la diffusion de documents scientifiques de niveau recherche, publiés ou non, émanant des établissements d'enseignement et de recherche français ou étrangers, des laboratoires publics ou privés. 


\title{
A Time-Of-Flight Gamma Camera Data Acquisition System for Hadrontherapy Monitoring
}

\author{
X. Chen, B. Carlus, C. Caplan, L. Caponetto, J.-P. Cachemiche, S. Curtoni, D. Dauvergne, R. \\ Della-Negra, M. Fontana, L. Gallin-Martel, M.-L. Gallin-Martel, J. Hérault, D. Lambert, G.-N. Lu, M. \\ Magne, H. Mathez, C. Morel, G. Montarou, M. Rodo, E. Testa, Y. Zoccarato
}

\begin{abstract}
A collimated gamma camera coupled to a beam hodoscope is currently under development within the CLaRyS collaboration to provide ion-range verification during hadrontherapy. We propose a $\mu \mathrm{TCA}$-based data acquisition (DAQ) system that consists of dedicated front-end boards, a multi-optical DAQ board, a reference clock board and a computer. Several specific developments have been performed in the front-end boards, namely a TDC (time-to-digital converter) based system synchronization, an adjustable-depth measureddata buffer, an histogram-based fast time-of-flight estimation and an easy adaptation for different applications. All these DAQ developments have been recently tested with a reduced-size hodoscope and collimated camera in the Mediterranean Protontherapy Institute in Nice.
\end{abstract}

Index Terms - Collimated $\gamma$-Camera, DAQ, TDC, prompt gamma, hadrontherapy verification

\section{INTRODUCTION}

$S^{\mathrm{e}}$ everal methods have been proposed to perform ion-range verification for Quality Assurance in hadrontherapy. Prompt gamma detection is one of them, and has lead to the development of two gamma camera prototypes, coupled with a beam hodoscope upstream of the patient (in a French research collaborative project involving IPNL Lyon, LPSC Grenoble, CPPM Marseille, CREATIS Lyon): a collimated camera (with multi-parallel slit collimator) and a Compton camera (CC)[1].

The hodoscope consists of two crossed arrays of scintillating

This work was partially performed in the framework of FP7-ENTERVISION network (Grant Agreement number 264552), FP7-ENVISION programmer (Grant Agreement number241851), LabEx PRIMES ANR-11-LABX-0063/ANR-11-IDEX-0007 and ITMO-Cancer (CLaRyS-UFT project).

X. Chen, B. Carlus, L. Caponetto, R. Della-Negra, M. Fontana, H. Mathez, E. Testa and Y. Zoccarato are with the Institute of Nuclear physics of Lyon (IPNL), University of Lyon 1, IN2P3/CNRS, UMR 5822, F-69622 Villeurbanne cedex, France (e-mail: x.chen@ipnl.in2p3.fr).

C. Caplan, J.-P. Cachemiche, C. Morel and M. Rodo are with the Aix-Marseille Univ, IN2P3/CNRS, CPPM, Marseille,, France.

S. Curtoni, D. Dauvergne, L. Gallin-Martel and M.-L. Gallin-Martel are with Laboratory of Subatomic Physics \& Cosmology, Grenoble-Alpes University, IN2P3/CNRS, UMR 5821, Grenoble.

J. Hérault is with Centre Antoine Lacassagne, Cyclotron Biomédical, 227 Avenue de la Lanterne, 06200 Nice, France

G.-N. Lu is with Institute of Nanotechnologies of Lyon (INL), University of Lyon 1, UMR CNRS 5270, F-69622 Villeurbanne cedex, France.

D. Lambert, M. Magne and G. Montarou are with LPC, Blaise Pascal university, IN2P3/CNRS, F-63178, Aubière cedex, France. fibers to allow for spatial and time tagging of incident ions. Spatial information is used for image reconstruction, and temporal information allowing time-of-flight (TOF) measurement serves to reduce neutron background and thus to enhance signal-to-noise ratio. The two gamma camera prototypes have been designed to employ the same absorber consisting of BGO (Bismuth Germanate) blocks.

Both prototypes require a data acquisition (DAQ) system with the main following specifications: $1 \mu$ s trigger delay for Compton camera, time resolution better than $1 \mathrm{~ns}, \sim 100$ and $500 \mathrm{kHz}$ trigger rate for collimated camera and Compton camera respectively, and $\sim 500 \mathrm{Mbits} / \mathrm{s}$ final storage rate.

We present here a DAQ system designed for this purpose. It has recently been tested with a reduced-size hodoscope and collimated camera in the Mediterranean Protontherapy Institute in Nice.

\section{ARCHITECTURE AND OPERATION}

The designed DAQ system mainly consists of the following parts (shown in Figure 1):

- Two 64-channel Hodoscope Front-End Readout (HFER) boards [2] for 64-channel Reduced-Size (RS) hodoscope,

- Six 24-channel absorber front-end readout boards (called ASM) [3] for thirty-six 4-pixel BGO block,

- A Micro Telecommunications Computing Architecture ( $\mu$ TCA)-based DAQ system mainly consisting of an Advanced Mezzanine Card board (AMC40) [2] and a PC for data acquisition, slow control and monitoring

- A 40-MHz reference clock and reset generator [2].

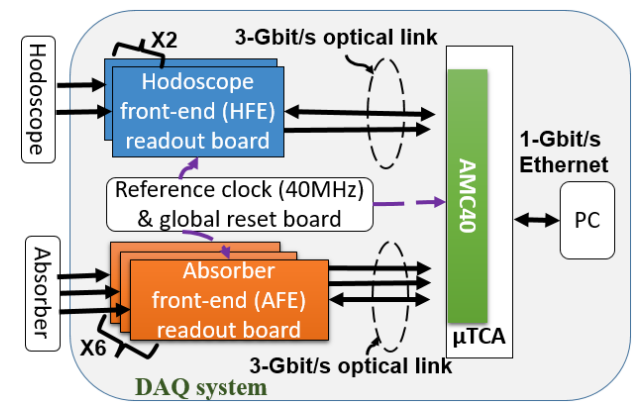

Figure 1. Scheme of the DAQ system.

\section{A. System description}

Both HFER and ASM boards (front-end boards) perform position determination and time of arrival (TOA) measurement. In addition, the ASM board allows signal charge measurement. 
The AMC40 board serves as data transfer station for every DAQ components except the clock-and-reset generator board. It connects to the front-end boards via a $3 \mathrm{Gbit} / \mathrm{s}$ signal-channel optical link (up to 36 links available) and connects to the PC through a 1 Gbits/s Ethernet link.

The DAQ system operates as follows: when a prompt gamma (PG) is detected in a BGO block, its associated ASM board issues a trigger signal to every HFER board for coincidence event searching. Coincidence events correspond to hodoscope and trigger events located within a TOF window centered on the PG TOA. The front-end boards send the data to the PC for data storage.

These boards incorporates several multi-phase time-to-digital converters (MPTDC): one for tagging the global reset signal and the other ones for tagging the detected signal. The final detected-signal's timestamp is obtained by its measured timestamp minus reset-signal's timestamp. Compared to conventional synchronization methods resetting coarse time which leads to potential meta-stability of one clock tick, this method can reduce reset error to 1 least-significant bit (LSB) of TDC.

\section{B. Frond-end boards description}

The front-end boards have the same basic structure, consisting of a FPGA, an optical transceiver, a clock jitter cleaner and a multi-channel analog readout module. The main difference between the two boards is that the former incorporates two 32-channel readout ASICs, and the later employs 8-channel DRS4 (Domino Ring Sampling) mezzanines (3 for each board).

In addition to the three MP-TDCs with 312.5 ps LSB, the firmware implementation of HFER board consists of a specific adjustable-depth buffer for the measured data and a histogram-based fast TOF estimation, which is adapted for different applications and eases fast system debugging. It includes also a coincidence-event searching, zero-suppression for useful data and NIOSII-based slow control.

Besides four MP-TDC with $625 \mathrm{ps} \mathrm{LSB}$, the firmware implementation of ASM board also consists of a charge measurement as well as NIOSII-based slow control.

\section{BEAM TESTS}

The reduced-size multi-collimated camera system has been tested in September 2018, with the $65 \mathrm{MeV}$ proton beam line. The setup consists of a RS hodoscope associated to one HFER board, six BGO absorber blocks associated to one ASM board, a PMMA target and the $\mu$ TCA readout system. The experiment was performed as follows: this proton passed through RS hodoscope and then hit a PMMA target where PG were emitted. The PG passing through the collimator were then absorbed by a BGO block.

Another beam-test has been performed in March 2019 to evaluate the performance of the HFER readout board. The main difference in respect to the previous setup is that this setup was composed of two plastic scintillators located upstream and downstream of the RS hodoscope to provide the trigger signal (coincidence of the two plastics).

\section{RESULTS AND DISCUSSION}

For the first beam test, two different measurements with the target shifted by $1 \mathrm{~cm}$ along the beam direction were completed with a trigger rate ranging from 300 to $400 \mathrm{kHz}$. The final storage rate was about $200 \mathrm{Mbits} / \mathrm{s}$ after sending all the data to a PC. The mono-dimensional interaction position profiles along the beam direction are shown on Fig 2.a: the red curve corresponds to the initial position while the blue one corresponds to the shifted position. The maximum is closely related to the Bragg peak position, a few millimeters upstream. A shift of 2-3 bins is qualitatively visible between the two PG profiles. PG profiles will be improved by fine tuning the BGO blocks and ASM board parameters.
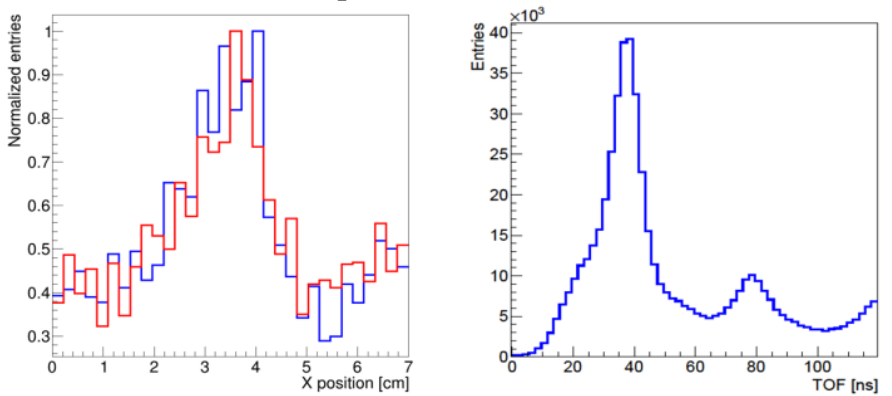

Figure 2: a) Mono-dimensional interaction position profile along the beam direction obtained for two acquisitions with the PMMA target set in two different positions; b) TOF spectrum obtained.

The TOF distributions (Fig 2.b) shows the beam time structure, with bunches delivered every $40 \mathrm{~ns}$. Two bunches are clearly visible in the presented distribution, and a third one is cut by the end of the coincidence window. The main peak (close to $40 \mathrm{~ns}$ ) corresponds to the bunch that has caused the emission of the PG inducing the trigger signal.

The second beam test was performed with several beam intensities resulting in data rate from $13 \mathrm{kHz}$ to $30 \mathrm{MHz}$. The measured efficiency was about $75 \%$ for data rate lower than $13 \mathrm{MHz}$ and slightly decreases down to $65 \%$ at $30 \mathrm{MHz}$.

\section{CONCLUSION}

The proposed DAQ system adopted several special techniques for an easy adaptation to different applications. The beam tests show that the system can operate with $312.5 \mathrm{~ns}$ LSB timing resolution, a storage rate of $\sim 200 \mathrm{Mbits} / \mathrm{s}$ for a count rate of $400 \mathrm{kHz}$. Another beam test for the full version of the collimated camera coupled to a RS hodoscope is in preparation.

\section{REFERENCES}

[1] J. Krimmer, D. Dauvergne, J. M. Létang, E. Testa, Prompt-gamma monitoring in hadrontherapy: A review, Nuclear Instruments and Methods in Physics Research Section A: Accelerators,Spectrometers, Detectors and Associated Equipment. https://doi.org/10.1016/j.nima.2017.07.063(2017)

[2] X. Chen et al., "A Data Acquisition System for a Beam-Tagging Hodoscope used in Hadrontherapy Monitoring," 2017 IEEE Nuclear Science Symposium and Medical Imaging Conference (NSS/MIC), Atlanta, GA, 2017, pp. 1-4. doi: 10.1109/NSSMIC.2017.8532646

[3] Arnaud Rozes. Construction et premières caractérisations d'un détecteur dédié à la mesure de l'activité $\beta+$ induite lors des traitements d'hadronthérapie, en vue de leur contrôle balistique. Université Blaise Pascal - Clermont-Ferrand II, 2016. Français. 